\title{
THE PATHOLOGICAL MORPHOLOGY OF PIROPLASMOSIS IN CATTLE
}

\author{
Aizhan Balgimbaeva ${ }^{1}$, Gulnar Schabdarbaeva ${ }^{2}$, Assem Ibazhanova ${ }^{3}$, Zhuldyzay \\ Kenzhebekova $^{4}$
}

\begin{abstract}
Piroplasmosis in cows manifests mainly as a violation of erythrocytes, degenerative changes in parenchymal organs, general jaundice, hemosiderosis, and splenic hyperplasia. First, it affects the liver, followed by a violation of the digestive system; then, kidney function is impaired resulting in a breach of toxic substance released from the body. The blood is depleted in the number of erythrocytes, leukocytes, and hemoglobin. From decaying under the influence of parasite, hemoglobin is released from the red blood cells and is partially excreted by the kidneys in urine, resulting in the urine becoming dark red in color (hence, the disease is colloquially called "blood urine"). Hemoglobin part is processed into bile pigments; however, visible mucous membranes (eyes and mouth), skin, and subcutaneous tissue become yellowish.
\end{abstract}

UDC Classification: 619:576.895.42-0.92:0.53.2, DOI: http://dx.doi.org/10.12955/cbup.v3.633

Keywords: Piroplasma, cattle, pathology, morphology, histology, bloody urine, coastal rush

\section{Introduction}

Piroplasmosis of cattle (Texas fever) is a transmissible animal disease caused by parasites of the red blood cells. Piroplasma bigeminum is an endoglobulyar parasite causing piroplasmoses (Dorosh, 2007).

Several common names describe the clinical signs of this disease, including: "“Bloody urine", "Chihir", "Coastal Rush", and "Texas fever". The life cycle of organisms causing piroplasmosis have two main vectors, cattle and ticks. Piroplasmosis reproduction in animals occurs in the blood, by simply dividing. In ticks it reproduces sexually in tissues, eggs and the hemolymph. In the body of animals, the parasites first infest internal organs, and then the peripheral blood (Stepanova; 1982; Dorosh, 2007).

In places, piroplasmoses are widespread with development of intensive livestock production and create considerable difficulties. Imported purebred animals affected by piroplasmosis, in most cases, die. This lost productivity causes livestock operations to become uneconomical (Shabdarbayeva, 2008).

Piroplasmosis in cattle is distributed mainly in the southern region of Kazakhstan. Here it proceeds benignly compared to warmer areas. Outbreaks occur in spring, summer, and in fall, sometimes (Sabanshiev, Suleimenov, Karamendin, Shabdarbayeva, \& Zhantore, 2003).

\section{Materials and Methods}

This study was conducted in the Kazakh National Agrarian University in the Department of Biological Safety. Material for research involved the internal organs from five carcasses of cows, imported from the Kainar rural district, Almaty. In addition, the study used internal organs of two cows that were experimentally infected with blood strains isolated from spontaneously infected cows by Piroplasma bigeminum, resulting in piroplasmosis.

A pathanatomical report was completed with the cows' autopsy.

The histological examination involved taking pieces of internal organs: liver, kidneys, heart, spleen, lungs, stomach, small and large intestines, and brain.

A $4-5 \mathrm{~cm}$ piece of approximately $0.5 \mathrm{~cm}$ thickness was taken from all organs. Materials were fixed in $10 \%$ neutral formalin in Carnoy's fluid. Pieces were compacted in celloidin-paraffin, and then frozen.

\footnotetext{
${ }^{1}$ Aizhan Balgimbaeva, Kazakh National Agrarian University, balgimbaeva@mail.ru

${ }^{2}$ Gulnar Schabdarbaeva , Kazakh National Agrarian University, Shgs52@mail.ru

${ }^{3}$ Assem Ibazhanova, Kazakh National Agrarian University, sema.serikova@mail.ru

${ }^{4}$ Zhuldyzay Kenzhebekova, Kazakh National Agrarian University, juldiz_03@mail.ru
} 
Thin slices were sectioned from compacted pieces using a semiautomatic microtome (ERM 3100), and then stained with hematoxylin-eosin to produce the Romanovsky - Geimsa effect for a scoping study of cell differentiation.

\section{Results}

Clinical examination of the cattle revealed general weakness, temperature $40-41^{\circ} \mathrm{C}$, decreased appetite, increased heart rate and breathing, anemia, and yellowness of the visible mucous membranes. Careful examination of the body of cattle revealed ticks, which were removed. Many clinical signs were typical of cattle piroplasmosis.

Macroscopic picture: On gross examination of carcasses, yellowness of the sclera, mucous membranes (pleura, peritoneum) and internal organs were observed (Figures 1 and 2). In addition to this was swelling of the dewlap, and belly. There was multiple bleeding (hemorrhagic diathesis) in serous, mucous membranes and internal organs, and yellowness indicating anemia. The blood was "watery" and not able to clot.

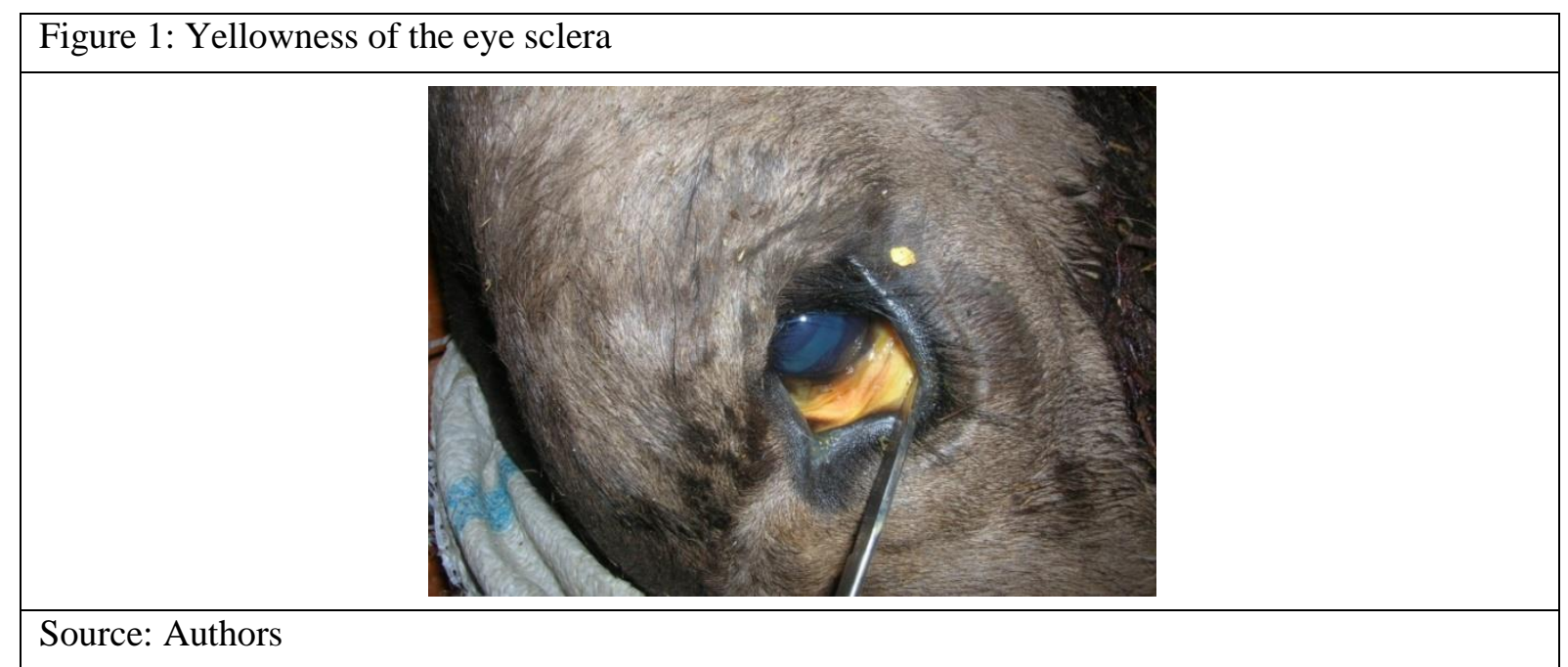

\begin{tabular}{|l|}
\hline Figure 2: Yellowness of the mucous membranes of the peritoneum \\
\hline
\end{tabular}

The spleen was enlarged $2-3$ times (a phenomenon called splenmegalies), with rounded edges on the cut-pulp protuberans, a soft consistency, and pulp scraping, indicating a marked phenomenon of septic spleen, which is characteristic of piroplasmosis (Figures 3 and 4). 
CBU INTERNATIONAL CONFERENCE ON INNOVATION, TECHNOLOGY TRANSFER AND EDUCATION MARCH 25-27, 2015, Prague, CZECH REPUBLIC
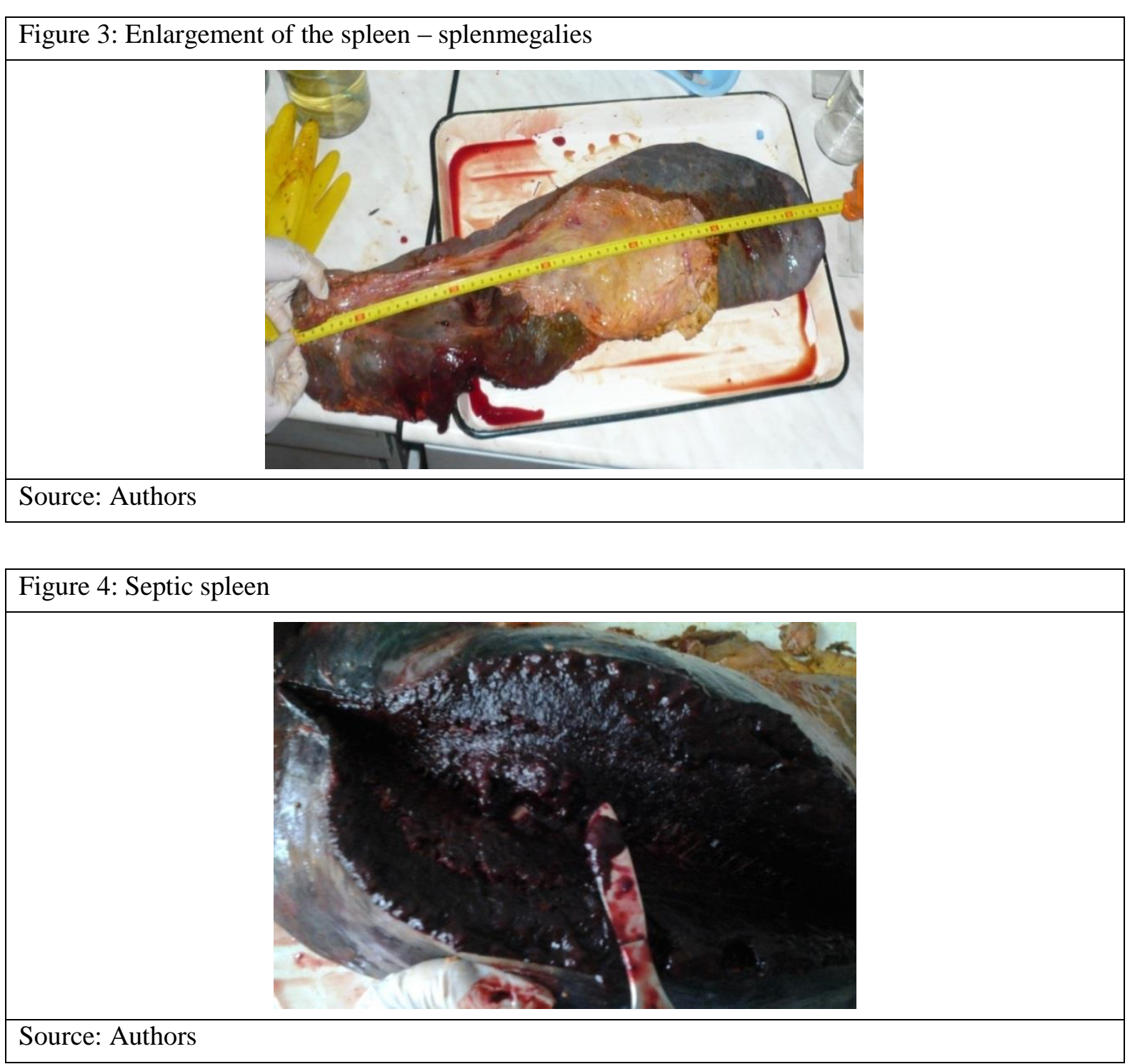

The liver was enlarged, with a flabby consistency, brownish-yellow in color, and with a "Clary species" appearance. These were attributed to degenerative processes and congestive hyperemia. The gall bladder was crowded with thick dark brown liquids. The liver's red-yellow doughy consistency on the cut surface had a deep red color under the pleura, with foci of hemorrhage (Figures 5 and 6).

\begin{tabular}{|l|}
\hline Figure 5: Increase overflow and gall bladder \\
\hline
\end{tabular}




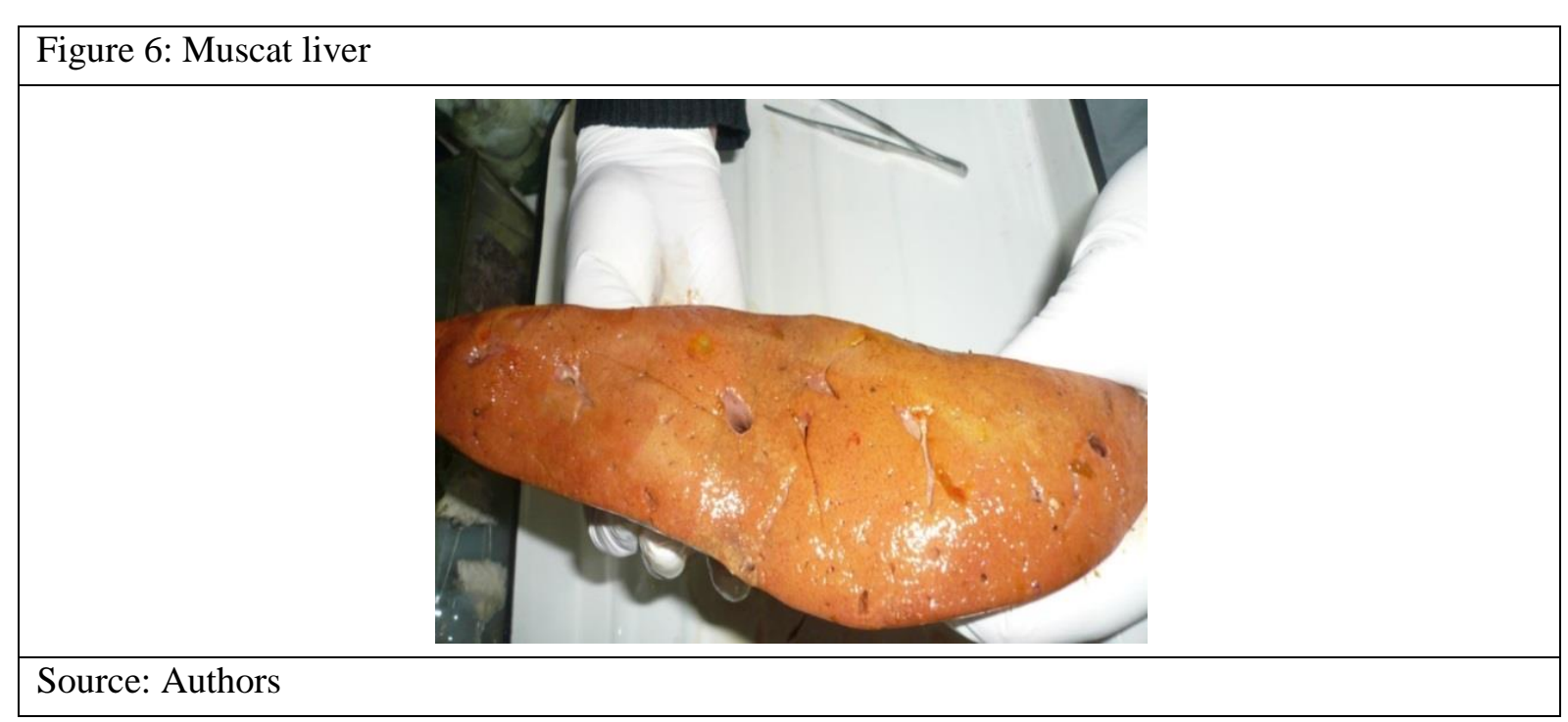

Buds were reddish-brown, flabby, and in the cortical layer petechiae had medulla that were radially striated, due to congestion of the capillaries.

The bladder comprised a transparent reddish liquid, within a thickened wall, with hemorrhages, which are characteristic of piroplasmosis (bloody urine).

The heart was enlarged due to the expansion of its cavities, especially of the right ventricle, and the atrial myocardium was flabby and dull.

There were enlarged lymph nodes that appeared "juicy", and hyperemic.

Skeletal muscles were flabby, dull, and a brownish-red color.

An enlarged omasum (the "book") contained dense dry fodder weight, indicating the phenomenon of proventriculus atony (Figure 7).

The mucous membranes of the abomasum for the extent of the intestines had hemorrhages, covered with a thick mucus mass.

Meningeal vessels were filled with blood.

\begin{tabular}{|l|l|}
\hline Figure 7: Atony phenomena in the omasum ("book") \\
\hline Source: Authors
\end{tabular}

Histological changes. In the spleen, the histological section was marked red-pulp hypoplasia, sometimes completely filling the space. Generally, there were large cells with nuclei and a prominent loose rim of cytoplasm, macrophages, rod and segmented neutrophils, reticular cells, and megacaryocytes. The 
macrophages contained the brown pigment hemosiderin. The trabeculae of the spleen was dense, and intensely colored.

The structure of the liver lobules showed loss of beams. The nucleus of hepatocytes had poor chromatin in the cytoplasm with dust-like brownish to pink grains or vacuoles with delicate mesh. These are signs that indicate granular and vacuolar degeneration. Beams located between the liver cells and lymphoid cells were apparent. A connective tissue layer was infiltrated with lymphocytes, monocytes, and neutrophils. The content of blood vessels involved a network of deformed red blood cells, and fibrin clot or tightening of the vessels.

Tubules were unclear and delineated with large casements. Epithelial cells did not have clear boundaries, and were cloudy, either with pale blue nuclei or without nuclei. There were also intensely colored tubules, in which cells were small with a dense nucleus, in two cores. In some glomeruli, there was marked hyperemia and endothelial dystrophy, and others bionecrosis at the center, and proliferation of oval cells at the periphery.

Myocardial dystrophy was indicated by muscle fibers without clear boundaries, a murky core, and uneven coloring of the eosin.

Lumens of alveoli were clean; with some having an outgrowth of cells with loose light-blue nuclei, and the cytoplasm of alveolar macrophages had a muddy appearance. Some parts of the interalveolar septum were thinned, and interrupted, while in other places, these were thickened, and infiltrated with lymphoid and epithelioid cells. Thickened blood vessels were also present. Bronchial epithelium had a cloudy swelling, and contained a thin surface of cloyocifils mucus.

There was marked degeneration of the epithelial cells of the intestine, and this was apparent with the lobular epithelium metaplasia in goblet cells. In the crypts of many cells were thick, bright, villi, which became paler nearer the surface. Submucosa villi were loose in some places and contained accumulations of epithelioid cells.

In the friable brain, substance was homogeneous oxyphilic material. In neurocyte and glial cells were marked pericellular edema, although there are also little change in neurocytes, with intact outgrowth. Blood vessels were distended with blood.

\section{Conclusion}

Thus, the pathoanatomical and histological study of seven carcasses of cattle, brought from Almaty region, and experimentally infected, showed almost all characteristics of piroplasmosis in terms of pathological and histological changes. These were in the form of hemosiderosis of the liver, spleen, kidneys, granular and fatty degeneration of parenchymal organs, and proliferation of histiocytes and lymphoid cells. In the spleen, there was an abundance of red blood cells in the red pulp, with weak white pulp (follicles). In the liver, apart from the hemosiderosis and granular dystrophy, were marked necrobiosis of cells of the central portion of lobules (muscat), perivascular edema, with diffuse and focal proliferation of lymphoid cells, and histiocytes. In the brain was necrosis and lysis of ganglion cells, chromotrolisis, hyperemia, and hemorrhage.

\section{References}

Dorosh, M. (2007). Diseases of cattle. Moscow:Veche.

Sabanshiev, M., Suleimenov, T., Karamendin, O., Shabdarbayeva, \& G., Zhantore, M. (2003). Parasitology and invasive diseases of animals. Almaty: Publishing house of Agrouniversity.

Shabdarbayeva, G.S. (2008). Immunobiological aspects of designing anti-idiotype-based diagnostics to identify blood parazites. (Doctoral Dissertation) Kazakh National Agrarian University, Almaty.

Stepanova, N. I. (Ed.) (1982). Protozoan disease of farm animals. Moscow: Kolos. 